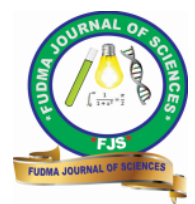

FUDMA Journal of Sciences (FJS)

ISSN online: 2616-1370

ISSN print: 2645 - 2944

Vol. 4 No. 3, September, 2020, pp $563-575$

DOI: https://doi.org/10.33003/fjs-2020-0403-326

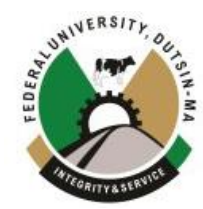

\title{
A NEW FOUR-PARAMETER WEIBULL DISTRIBUTION WITH APPLICATION TO FAILURE TIME DATA
}

\author{
*1 Aminu Suleiman Mohammed and ${ }^{2}$ Fidelis Ifeanyi Ugwuowo \\ ${ }^{1}$ Department of Statistics, Ahmadu Bello University, Zaria, Kaduna State, Nigeria. \\ ${ }^{2}$ Department of Statistics, University of Nigeria, Nsukka, Enugu State, Nigeria. \\ ${ }^{*}$ Corresponding Author's Email: mohammedas@abu.edu.ng
}

\begin{abstract}
A lifetime model called Transmuted Exponential-Weibull Distribution was proposed in this research. Several statistical properties were derived and presented in an explicit form. Maximum likelihood technique is employed for the estimation of model parameters, and a simulation study was performed to examine the behavior of various estimates under different sample sizes and initial parameter values. Through using reallife datasets, it was empirically shown that the new model provides sufficient fits relative to other existing models.
\end{abstract}

Keywords: Weibull distribution, Reliability function, Maximum Likelihood, Order statistics.

\section{INTRODUCTION}

There are a variety of continuous distributions that are used to model lifetime data, such as Weibull, exponential, Lindley and gamma among other distributions (Shanker et al., 2015). One of the famous distribution named after the Swedish physicist Waladdi Weibull is the Weibull distribution (Murthy et al., 2004). In 1939 he used this model to measure the strength of material breakage. The distribution has two parameters that make it a flexible model that can take on the characteristics of many other distributions. Weibull distribution, however, plays a critical role in life research which is comparable to other distributions in other statistical fields. Also, it does not have a sufficient fit for modeling failure rates with bathtub and unimodal form in reliability and other relevant areas. The statistical analysis is based exclusively on the assumed probability distribution. Despite this, substantial efforts have been made to construct families of probability distributions that are enriched in statistical methodologies.

In the light of statistical literature, some extensions of the Weibull model can be obtained, such as the additive Weibull model with failure rate function having bathtub shaped by (Xie and Lai 1995), Mudholkar et. al. (1996) proposed a generalization of the Weibull distribution with uses on the survival data, extended Weibull by (Xie et al. 2002), Parameters estimation of the modified Weibull distribution by (Zaindin and Sarhan 2009), Aryal andTsokos (2011) proposed Transmuted Weibull distribution, Cordeiro et. al. (2014) developed McDonald Weibull model, Calderín-Ojeda (2015). Studied the Composite Weibull-Burr Model to describe claim data, Ahmad et. al. (2017) studied the structural properties of Weibull-Rayleigh distribution with uses to lifetime dataset, Cordeiro et. al. (2018) proposed Lindley Weibull distribution. Some other relevant researches include Daniyal \& Aleem (2014) studied the mixture of Burr XII and Weibull distributions, Mohammed (2019) studied theoretical analysis of the Exponentiated Transmuted Kumaraswamy Distribution, On the structural properties and applications of APT class of distributions by Mead et al. (2019), Mohammed and Yahaya (2019) studied Exponentiated Transmuted Inverse Exponential Distribution, Fréchet-Weibull Distribution with Applications to Earthquakes Datasets by (Teamah et al, 2020).

Recently, in the context of literature, numerous families of probability models have been studied and it has been proven that these distributions give better fits than the baseline distributions. These classes comprise the T-X class by Alzaatreh et al. (2013), the Exp-G (EG) class by Cordeiro et al. (2013), the Lom-G class by Cordeiro et al. (2014), the Wei-G class by Bourguignon et al. (2014) and A new family of distributions for generating skewed distributions by (Mohammed \& Ugwuowo 2020).

Here, a new four-parameter model was introduced, called Transmuted Exponential-Weibull Distribution. Regarding remaining parts of this article, the following arrangement is considered. In section 2, along with many of its characteristics, we presented the proposed model 's cumulative distribution function (cdf) and probability density function (pdf). The entropy and order statistics of the proposed model are provided in section 3. In section 4, the estimation of the proposed parameters of the model was carried out using the maximum likelihood technique. Section 5 presents a simulation analysis under the methodology of maximum - likelihood estimation for the unknown distribution parameters. Together with other related distributions, the proposed distribution is applied to real-life datasets in section 6. Eventually, the paper ends in section 7 .

\section{Transmuted Exponential- Weibull (TE-W) Model}

For a continuous random variable say $\mathrm{X}$, the cumulative distribution function (cdf) and probability density function (pdf) of the TE- G family of distributions (Mohammed and Ugwuowo 2020) are respectively given by; 
$F(x ; \lambda, \theta, \xi)=\left(1-(1-G(x, \xi))^{\lambda}\right)\left(1+\theta(1-G(x, \xi))^{\lambda}\right)$

and

$$
f(x ; \lambda, \theta, \xi)=\frac{g(x, \xi)}{1-G(x, \xi)} \lambda(1-G(x, \xi))^{\lambda}\left(1-\theta+2 \theta(1-G(x, \xi))^{\lambda}\right)
$$

Where, $G(x, \xi)$ and $g(x, \xi)$ are the parent (baseline) cumulative distribution function (cdf) and probability density function (pdf) respectively depending upon a parameter vector $\xi$ and $\lambda>0,-1 \leq \theta \leq 1$ are two additional parameters i.e scale and transmuted parameter respectively.

\section{Definition of the new model}

Consider the density function $g(x ; k, \gamma)=\frac{k}{\gamma}\left(\frac{x}{\gamma}\right)^{k-1} e^{-\left(\frac{x}{\gamma}\right)^{k}}$ and distribution function $G(x ; k, \gamma)=1-e^{-\left(\frac{x}{\gamma}\right)^{k}}$ of the

Weibull distribution having scale $\gamma>0$ and shape $k>0$ parameters. Inducing the functions in (1) and (2), the cumulative distribution function (cdf) and probability density function (pdf) of Transmuted Exponential -Weibull Distribution (TE-WD) are respectively given as;

Definition 1. A random variable say $X$ is said to follow a Transmuted Exponential- Weibull distribution if its distribution and density function has the following form;

$F(x ; \lambda, \theta, k, \gamma)=\left(1-e^{-\lambda\left(\frac{x}{\gamma}\right)^{k}}\right)\left(1+\theta e^{-\lambda\left(\frac{x}{\gamma}\right)^{k}}\right)$

and

$f(x ; \lambda, \theta, k, \gamma)=\frac{\lambda k(1-\theta)}{\gamma}\left(\frac{x}{\gamma}\right)^{k-1} e^{-\lambda\left(\frac{x}{\gamma}\right)^{k}}+\frac{2 \lambda \theta k}{\gamma}\left(\frac{x}{\gamma}\right)^{k-1} e^{-2 \lambda\left(\frac{x}{\gamma}\right)^{k}}$

Where, $\lambda, k, \gamma>0$ and $|\theta| \leq 1$

Model Validity Check

Proposition 1: The TE-WD is a well valid probability density function

$\int_{0}^{\infty} f(x ; \lambda, \theta, k, \gamma) d x=1$

$\int_{0}^{\infty}\left\{\frac{\lambda k(1-\theta)}{\gamma}\left(\frac{x}{\gamma}\right)^{k-1} e^{-\lambda\left(\frac{x}{\gamma}\right)^{k}}+\frac{2 \lambda \theta k}{\gamma}\left(\frac{x}{\gamma}\right)^{k-1} e^{-2 \lambda\left(\frac{x}{\gamma}\right)^{k}}\right\} d x=1$

$\int_{0}^{\infty} \frac{\lambda k(1-\theta)}{\gamma}\left(\frac{x}{\gamma}\right)^{k-1} e^{-\lambda\left(\frac{x}{\gamma}\right)^{k}} d x+\int_{0}^{\infty} \frac{2 \lambda \theta k}{\gamma}\left(\frac{x}{\gamma}\right)^{k-1} e^{-2 \lambda\left(\frac{x}{\gamma}\right)^{k}} d x$

$\frac{\lambda k(1-\theta)}{\gamma^{k}} \int_{0}^{\infty} x^{k-1} e^{-\lambda\left(\frac{x}{\gamma}\right)^{k}} d x+\frac{2 \lambda \theta k}{\gamma^{k}} \int_{0}^{\infty} x^{k-1} e^{-2 \lambda\left(\frac{x}{\gamma}\right)^{k}} d x$

Recall that

$\int_{0}^{\infty} x^{n} e^{-a x^{b}} d x=\frac{1}{b} a^{-\frac{(n+1)}{b}} \Gamma\left(\frac{n+1}{b}\right)$ 
$\therefore \int_{0}^{\infty} x^{k-1} e^{-\frac{\lambda}{\gamma^{k}} x^{k}} d x=\frac{1}{k}\left(\frac{\lambda}{\gamma^{k}}\right)^{-\frac{k}{k}} \Gamma\left(\frac{k}{k}\right)=\frac{\gamma^{k}}{\lambda k} \times 1=\frac{\gamma^{k}}{\lambda k}$

and

$\int_{0}^{\infty} x^{k-1} e^{-\frac{2 \lambda}{\gamma^{k}} x^{k}} d x=\frac{1}{k}\left(\frac{2 \lambda}{\gamma^{k}}\right)^{-\frac{k}{k}} \Gamma\left(\frac{k}{k}\right)=\frac{\gamma^{k}}{2 \lambda k} \times 1=\frac{\gamma^{k}}{2 \lambda k}$

$\Rightarrow \frac{\lambda k(1-\theta)}{\gamma^{k}} \times \frac{\gamma^{k}}{\lambda k}+\frac{2 \theta \lambda k}{\gamma^{k}} \times \frac{\gamma^{k}}{2 \lambda k}=1-\theta+\theta=1$

Graphical illustration of the density and distribution function of TE-WD

The plots of the cumulative distribution function (cdf) and probability density function (pdf) of the TE-WD are respectively shown in figure 1 and 2 for selected values $\lambda=a, k=b, \gamma=c$, and $\theta=d$

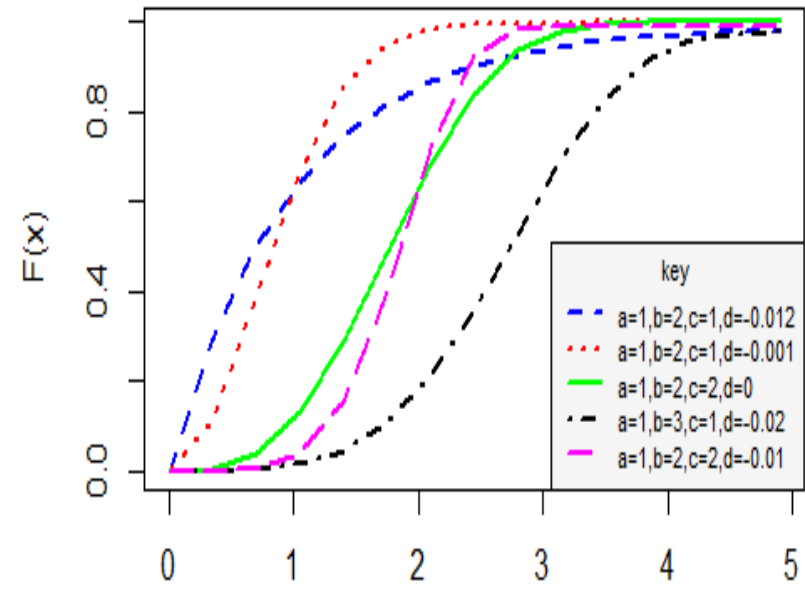

$x$

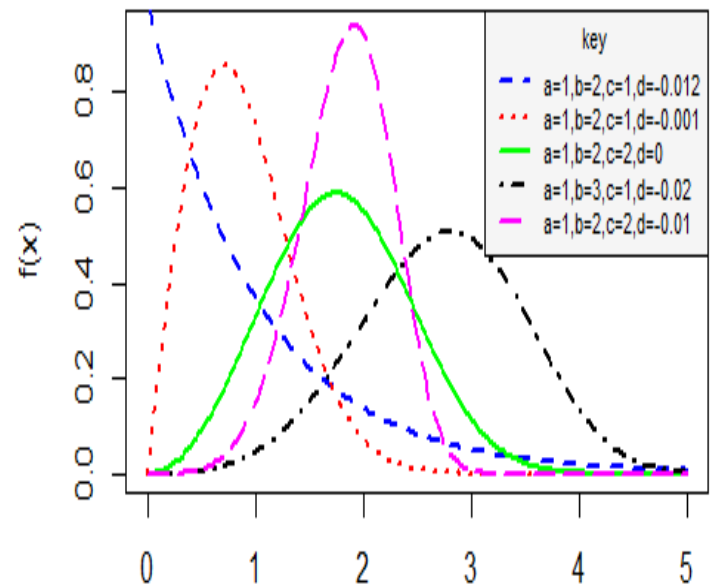

$x$

Figure 1: cdf plot of TE-WD

Figure 2: pdf plot of TE-WD

Statistical and Mathematical Properties of TE-WD

In this section, some properties of the TE-W distribution were presented. They include the following:

Moments and Moment generating function

Definition 2. Let $X$ be a random variable with the TE-WD defined in (4).

Proposition 2: The $\mathrm{r}^{\text {th }}$ Moments of TE-WD is given by;

$\mu_{r}^{\prime}=\frac{\gamma^{r} \Gamma\left(1+\frac{r}{k}\right)}{\lambda^{\frac{r}{k}}}\left(1-\theta+\frac{\theta}{2^{\frac{r}{k}}}\right)$

Proof:

$\mu_{r}^{\prime}=\int_{0}^{\infty} x^{r}\left\{\frac{\lambda k(1-\theta)}{\gamma}\left(\frac{x}{\gamma}\right)^{k-1} e^{-\lambda\left(\frac{x}{\gamma}\right)^{k}}+\frac{2 \lambda \theta k}{\gamma}\left(\frac{x}{\gamma}\right)^{k-1} e^{-2 \lambda\left(\frac{x}{\gamma}\right)^{k}}\right\} d x$
$\mu_{r}^{\prime}=\int_{0}^{\infty} x^{r} \frac{\lambda k(1-\theta)}{\gamma}\left(\frac{x}{\gamma}\right)^{k-1} e^{-\lambda\left(\frac{x}{\gamma}\right)^{k}} d x+\int_{0}^{\infty} x^{r} \frac{2 \lambda \theta k}{\gamma}\left(\frac{x}{\gamma}\right)^{k-1} e^{-2 \lambda\left(\frac{x}{\gamma}\right)^{k}} d x$ 
$\mu_{r}^{\prime}=\frac{\lambda k(1-\theta)}{\gamma^{k}} \int_{0}^{\infty} x^{k+r-1} e^{-\frac{\lambda}{\gamma^{k}} x^{k}} d x+\frac{2 \lambda \theta k}{\gamma^{k}} \int_{0}^{\infty} x^{k+r-1} e^{-\frac{2 \lambda}{\gamma^{k} k^{k}}} d x$

Recall that;

$\int_{0}^{\infty} x^{n} e^{-a x^{b}} d x=\frac{1}{b} a^{-\frac{(n+1)}{b}} \Gamma\left(\frac{n+1}{b}\right)$

$\therefore \int_{0}^{\infty} x^{k+r-1} e^{-\frac{\lambda}{\gamma^{k}} x^{k}} d x=\frac{1}{k}\left(\frac{\lambda}{\gamma^{k}}\right)^{-\frac{k+r}{k}} \Gamma\left(\frac{k+r}{k}\right)=\frac{\gamma^{k} \gamma^{r}}{\lambda \lambda^{\frac{r}{k}} k} \Gamma\left(1+\frac{r}{k}\right)$

and

$\int_{0}^{\infty} x^{k+r-1} e^{-\frac{2 \lambda}{\gamma^{k}} x^{k}} d x=\frac{1}{k}\left(\frac{2 \lambda}{\gamma^{k}}\right)^{-\frac{k+r}{k}} \Gamma\left(\frac{k+r}{k}\right)=\frac{\gamma^{k} \gamma^{r}}{2 \times 2^{\frac{r}{k}} \lambda \lambda^{\frac{r}{k}} k} \Gamma\left(1+\frac{r}{k}\right)$

$\mu_{r}^{\prime}=\frac{\lambda k(1-\theta)}{\gamma^{k}} \times \frac{\gamma^{k} \gamma^{r}}{\lambda \lambda^{\frac{r}{k}} k} \Gamma\left(1+\frac{r}{k}\right)+\frac{2 \lambda \theta k}{\gamma^{k}} \times \frac{\gamma^{k} \gamma^{r}}{2 \times 2^{\frac{r}{k}} \lambda \lambda^{\frac{r}{k}} k} \Gamma\left(1+\frac{r}{k}\right)$

$\mu_{r}^{\prime}=\frac{\gamma^{r}(1-\theta) \Gamma\left(1+\frac{r}{k}\right)}{\lambda^{\frac{r}{k}}}+\frac{\theta \gamma^{r} \Gamma\left(1+\frac{r}{k}\right)}{2^{\frac{r}{k}} \lambda^{\frac{r}{k}}}$

$\mu_{r}^{\prime}=\frac{\gamma^{r} \Gamma\left(1+\frac{r}{k}\right)}{\lambda^{\frac{r}{k}}}\left(1-\theta+\frac{\theta}{2^{\frac{r}{k}}}\right)$

The corresponding moments for $\mathrm{r}=1,2,3,4$ are as follows;

If $r=1$

$\mu_{1}^{\prime}=\frac{\gamma^{1} \Gamma\left(1+\frac{1}{k}\right)}{\lambda^{\frac{1}{k}}}\left(1-\theta+\frac{\theta}{2^{\frac{1}{k}}}\right)$

If $r$ takes the value 2, then;

$\mu_{2}^{\prime}=\frac{\gamma^{2} \Gamma\left(1+\frac{2}{k}\right)}{\lambda^{\frac{2}{k}}}\left(1-\theta+\frac{\theta}{2^{\frac{2}{k}}}\right)$

If $r=3$

$\mu_{3}^{\prime}=\frac{\gamma^{3} \Gamma\left(1+\frac{3}{k}\right)}{\lambda^{\frac{3}{k}}}\left(1-\theta+\frac{\theta}{2^{\frac{3}{k}}}\right)$

If $r=4$

$\mu_{4}^{\prime}=\frac{\gamma^{4} \Gamma\left(1+\frac{4}{k}\right)}{\lambda^{\frac{4}{k}}}\left(1-\theta+\frac{\theta}{2^{\frac{4}{k}}}\right)$ 
For variance

$V(x)=\mu_{2}^{\prime}-\left(\mu_{1}^{\prime}\right)^{2}$

$\operatorname{Var}(x)=\frac{\gamma^{2} \Gamma\left(1+\frac{2}{k}\right)}{\lambda^{\frac{2}{k}}}\left(1-\theta+\frac{\theta}{2^{\frac{2}{k}}}\right)-\left(\frac{\gamma \Gamma\left(1+\frac{1}{k}\right)}{\lambda^{\frac{1}{k}}}\left(1-\theta+\frac{\theta}{2^{\frac{1}{k}}}\right)\right)^{2}$

Proposition 3: The $\mathrm{r}^{\text {th }}$ Moment about the Mean is given by;

$E(x-\mu)^{r}=\sum_{m=0}^{\infty}(-1)^{m}\left(\begin{array}{l}r \\ m\end{array}\right) \mu^{m}\left\{\frac{\lambda(1-\theta)}{\gamma^{k}}\left(\frac{\lambda}{\gamma^{k}}\right)^{-\left(\frac{r+k-m}{k}\right)}+\frac{2 \lambda \theta}{\gamma^{k}}\left(\frac{2 \lambda}{\gamma^{k}}\right)^{-\left(\frac{r+k-m}{k}\right)}\right\} \Gamma\left(\frac{r+k-m}{k}\right)$

Proof:

$E(x-\mu)^{r}=\int_{0}^{\infty}(x-\mu)^{r}\left\{\frac{\lambda k(1-\theta)}{\gamma}\left(\frac{x}{\gamma}\right)^{k-1} e^{-\lambda\left(\frac{x}{\gamma}\right)^{k}}+\frac{2 \lambda \theta k}{\gamma}\left(\frac{x}{\gamma}\right)^{k-1} e^{-2 \lambda\left(\frac{x}{\gamma}\right)^{k}}\right\} d x$

By applying Binomial expansion, we have,

$$
\begin{aligned}
& (x-\mu)^{r}=\sum_{m=0}^{\infty}(-1)^{m}\left(\begin{array}{c}
r \\
m
\end{array}\right) x^{r-m} \mu^{m} \\
& E(x-\mu)^{r}=\int_{0}^{\infty} \sum_{m=0}^{\infty}(-1)^{m}\left(\begin{array}{c}
r \\
m
\end{array}\right) x^{r-m} \mu^{m}\left\{\frac{\lambda k(1-\theta)}{\gamma}\left(\frac{x}{\gamma}\right)^{k-1} e^{-\lambda\left(\frac{x}{\gamma}\right)^{k}}+\frac{2 \lambda \theta k}{\gamma}\left(\frac{x}{\gamma}\right)^{k-1} e^{-2 \lambda\left(\frac{x}{\gamma}\right)^{k}}\right\} d x \\
& E(x-\mu)^{r}=\frac{\lambda k}{\gamma^{k}} \sum_{m=0}^{\infty}(-1)^{m}\left(\begin{array}{c}
r \\
m
\end{array}\right) \mu^{m} \int_{0}^{\infty}\left\{(1-\theta) x^{k+r-m-1} e^{-\lambda\left(\frac{x}{\gamma}\right)^{k}}+2 \theta x^{k-1} e^{-2 \lambda\left(\frac{x}{\gamma}\right)^{k}}\right\} d x
\end{aligned}
$$

Recall also,

$$
\begin{aligned}
& \int_{x=0}^{\infty} x^{n} e^{-a x^{b}} d x=\frac{1}{b} a^{-\left(\frac{n+1}{b}\right)} \Gamma\left(\frac{n+1}{b}\right) \\
& (1-\theta) \int_{0}^{\infty} x^{r+k-m-1} e^{-\frac{\lambda}{\gamma^{k}} k^{k}} d x=\frac{(1-\theta)}{k}\left(\frac{\lambda}{\gamma^{k}}\right)^{-\left(\frac{r+k-m}{k}\right)} \Gamma\left(\frac{r+k-m}{k}\right)
\end{aligned}
$$

and

$$
\begin{aligned}
& 2 \theta \int_{0}^{\infty} x^{k+r-m-1} e^{-\frac{2 \lambda}{\gamma^{k}} x^{k}} d x=\frac{2 \theta}{k}\left(\frac{2 \lambda}{\gamma^{k}}\right)^{-\left(\frac{r+k-m}{k}\right)} \Gamma\left(\frac{r+k-m}{k}\right) \\
& E(x-\mu)^{r}=\frac{\lambda k}{\gamma^{k}} \sum_{m=0}^{\infty}(-1)^{m}\left(\begin{array}{c}
r \\
m
\end{array}\right) \mu^{m}\left\{\frac{(1-\theta)}{k}\left(\frac{\lambda}{\gamma^{k}}\right)^{-\left(\frac{r+k-m}{k}\right)} \Gamma\left(\frac{r+k-m}{k}\right)+\frac{2 \theta}{k}\left(\frac{2 \lambda}{\gamma^{k}}\right)^{-\left(\frac{r+k-m}{k}\right)} \Gamma\left(\frac{r+k-m}{k}\right)\right\} \\
& E(x-\mu)^{r}=\frac{\lambda k}{\gamma^{k}} \sum_{m=0}^{\infty}(-1)^{m}\left(\begin{array}{c}
r \\
m
\end{array}\right) \mu^{m}\left\{\frac{(1-\theta)}{k}\left(\frac{\lambda}{\gamma^{k}}\right)^{-\left(\frac{r+k-m}{k}\right)}+\frac{2 \theta}{k}\left(\frac{2 \lambda}{\gamma^{k}}\right)^{-\left(\frac{r+k-m}{k}\right)}\right\} \Gamma\left(\frac{r+k-m}{k}\right)
\end{aligned}
$$




$$
E(x-\mu)^{r}=\sum_{m=0}^{\infty}(-1)^{m}\left(\begin{array}{l}
r \\
m
\end{array}\right) \mu^{m}\left\{\frac{\lambda(1-\theta)}{\gamma^{k}}\left(\frac{\lambda}{\gamma^{k}}\right)^{-\left(\frac{r+k-m}{k}\right)}+\frac{2 \lambda \theta}{\gamma^{k}}\left(\frac{2 \lambda}{\gamma^{k}}\right)^{-\left(\frac{r+k-m}{k}\right)}\right\} \Gamma\left(\frac{r+k-m}{k}\right)
$$

Corollary 1: if $\mu=0$, we have the moment about the origin as;

$$
E(x)^{r}=\frac{\gamma^{r} \Gamma\left(1+\frac{r}{k}\right)}{\lambda^{\frac{r}{k}}}\left\{(1-\theta)+\frac{\theta}{2^{\frac{r}{k}}}\right\}
$$

From the $\mathrm{r}^{\text {th }}$ moment about the origin If $\mathrm{r}=1,2,3$ and 4 we have,

$$
\begin{aligned}
& E(x-\mu)=\sum_{m=0}^{\infty}(-1)^{m}\left(\begin{array}{c}
1 \\
m
\end{array}\right) \mu^{m}\left\{\frac{\lambda(1-\theta)}{\gamma^{k}}\left(\frac{\lambda}{\gamma^{k}}\right)^{-\left(\frac{1+k-m}{k}\right)}+\frac{2 \lambda \theta}{\gamma^{k}}\left(\frac{2 \lambda}{\gamma^{k}}\right)^{-\left(\frac{1+k-m}{k}\right)}\right\} \Gamma\left(\frac{1+k-m}{k}\right) \\
& E(x-\mu)^{2}=\sum_{m=0}^{\infty}(-1)^{m}\left(\begin{array}{c}
2 \\
m
\end{array}\right) \mu^{m}\left\{\frac{\lambda(1-\theta)}{\gamma^{k}}\left(\frac{\lambda}{\gamma^{k}}\right)^{-\left(\frac{2+k-m}{k}\right)}+\frac{2 \lambda \theta}{\gamma^{k}}\left(\frac{2 \lambda}{\gamma^{k}}\right)^{-\left(\frac{2+k-m}{k}\right)}\right\} \Gamma\left(\frac{2+k-m}{k}\right) \\
& E(x-\mu)^{3}=\sum_{m=0}^{\infty}(-1)^{m}\left(\begin{array}{c}
3 \\
m
\end{array}\right) \mu^{m}\left\{\frac{\lambda(1-\theta)}{\gamma^{k}}\left(\frac{\lambda}{\gamma^{k}}\right)^{-\left(\frac{3+k-m}{k}\right)}+\frac{2 \lambda \theta}{\gamma^{k}}\left(\frac{2 \lambda}{\gamma^{k}}\right)^{-\left(\frac{3+k-m}{k}\right)}\right\} \Gamma\left(\frac{3+k-m}{k}\right) \\
& E(x-\mu)^{4}=\sum_{m=0}^{\infty}(-1)^{m}\left(\begin{array}{c}
4 \\
m
\end{array}\right) \mu^{m}\left\{\frac{\lambda(1-\theta)}{\gamma^{k}}\left(\frac{\lambda}{\gamma^{k}}\right)^{-\left(\frac{4+k-m}{k}\right)}+\frac{2 \lambda \theta}{\gamma^{k}}\left(\frac{2 \lambda}{\gamma^{k}}\right)^{-\left(\frac{4+k-m}{k}\right)}\right\} \Gamma\left(\frac{4+k-m}{k}\right)
\end{aligned}
$$

The coefficient of variation is therefore given as;

$$
\begin{aligned}
& C V=\frac{\sqrt{\sigma^{2}}}{\mu} \\
& C V=\frac{\lambda^{\frac{r}{k}} \sqrt{\sum_{m=0}^{\infty}(-1)^{m}\left(\begin{array}{c}
2 \\
m
\end{array}\right) \mu^{m}\left\{\frac{\lambda(1-\theta)}{\gamma^{k}}\left(\frac{\lambda}{\gamma^{k}}\right)^{-\left(\frac{2+k-m}{k}\right)}+\frac{2 \lambda \theta}{\gamma^{k}}\left(\frac{2 \lambda}{\gamma^{k}}\right)^{-\left(\frac{2+k-m}{k}\right)}\right\} \Gamma\left(\frac{2+k-m}{k}\right)}}{\gamma^{r} \Gamma\left(1+\frac{r}{k}\right)\left\{(1-\theta)+\frac{\theta}{2^{\frac{r}{k}}}\right\}}
\end{aligned}
$$

Coefficient of Skewness

$$
C S=\frac{E(x-\mu)^{3}}{\left(E(x-\mu)^{2}\right)^{\frac{3}{2}}}
$$




$$
C S=\frac{\sum_{m=0}^{\infty}(-1)^{m}\left(\begin{array}{c}
3 \\
m
\end{array}\right) \mu^{m}\left\{\frac{\lambda(1-\theta)}{\gamma^{k}}\left(\frac{\lambda}{\gamma^{k}}\right)^{-\left(\frac{3+k-m}{k}\right)}+\frac{2 \lambda \theta}{\gamma^{k}}\left(\frac{2 \lambda}{\gamma^{k}}\right)^{-\left(\frac{3+k-m}{k}\right)}\right\} \Gamma\left(\frac{3+k-m}{k}\right)}{\left(\sum_{m=0}^{\infty}(-1)^{m}\left(\begin{array}{c}
2 \\
m
\end{array}\right) \mu^{m}\left\{\frac{\lambda(1-\theta)}{\gamma^{k}}\left(\frac{\lambda}{\gamma^{k}}\right)^{-\left(\frac{2+k-m}{k}\right)}+\frac{2 \lambda \theta}{\gamma^{k}}\left(\frac{2 \lambda}{\gamma^{k}}\right)^{-\left(\frac{2+k-m}{k}\right)}\right\} \Gamma\left(\frac{2+k-m}{k}\right)\right)^{\frac{3}{2}}}
$$

Coefficient of Kurtosis

$$
\begin{aligned}
& C K=\frac{E(x-\mu)^{4}}{\left(E(x-\mu)^{2}\right)^{2}} \sum_{m=0}^{\infty}(-1)^{m}\left(\begin{array}{c}
4 \\
m
\end{array}\right) \mu^{m}\left\{\frac{\lambda(1-\theta)}{\gamma^{k}}\left(\frac{\lambda}{\gamma^{k}}\right)^{-\left(\frac{4+k-m}{k}\right)}+\frac{2 \lambda \theta}{\gamma^{k}}\left(\frac{2 \lambda}{\gamma^{k}}\right)^{-\left(\frac{4+k-m}{k}\right)}\right\} \Gamma\left(\frac{4+k-m}{k}\right) \\
& C K=\frac{\left(\sum_{m=0}^{\infty}(-1)^{m}\left(\begin{array}{c}
2 \\
m
\end{array}\right) \mu^{m}\left\{\frac{\lambda(1-\theta)}{\gamma^{k}}\left(\frac{\lambda}{\gamma^{k}}\right)^{-\left(\frac{2+k-m}{k}\right)}+\frac{2 \lambda \theta}{\gamma^{k}}\left(\frac{2 \lambda}{\gamma^{k}}\right)^{-\left(\frac{2+k-m}{k}\right)}\right\} \Gamma\left(\frac{2+k-m}{k}\right)\right)^{2}}{\left(\frac{2+k}{k}\right)}
\end{aligned}
$$

Proposition 4: the MGF of TE-WD is given as;

$$
M_{x}(t)=\sum_{r=0}^{\infty} \frac{t^{r} \gamma^{r} \Gamma\left(1+\frac{r}{k}\right)}{\lambda^{\frac{r}{k}} r !}\left(1-\theta+\frac{\theta}{2^{\frac{r}{k}}}\right)
$$

Proof:

$$
M_{x}(t)=E\left(e^{t x}\right)=\sum_{r=0}^{\infty} \frac{t^{r} E\left(X^{r}\right)}{r !}
$$

Where,

$$
\begin{aligned}
& E\left(X^{r}\right)=\frac{\gamma^{r} \Gamma\left(1+\frac{r}{k}\right)}{\lambda^{\frac{r}{k}}}\left(1-\theta+\frac{\theta}{2^{\frac{r}{k}}}\right) \\
& M_{x}(t)=\sum_{r=0}^{\infty} \frac{t^{r} \gamma^{r} \Gamma\left(1+\frac{r}{k}\right)}{\lambda^{\frac{r}{k}} r !}\left(1-\theta+\frac{\theta}{2^{\frac{r}{k}}}\right)
\end{aligned}
$$

Survival function (sf) of TE-WD

The sf of the model is given by;

$$
S(x)=\left(1-\theta+\theta e^{-\lambda\left(\frac{x}{\gamma}\right)^{k}}\right) e^{-\lambda\left(\frac{x}{\gamma}\right)^{k}}
$$

Hazard function (hf) of TE-WD

The hf of the model is given as; 
$h(x)=\frac{\left(\frac{\lambda k(1-\theta)}{\gamma}\left(\frac{x}{\gamma}\right)^{k-1}+\frac{2 \lambda \theta k}{\gamma}\left(\frac{x}{\gamma}\right)^{k-1} e^{-\lambda\left(\frac{x}{\gamma}\right)^{k}}\right)}{\left(1-\theta+\theta e^{-\lambda\left(\frac{x}{\gamma}\right)^{k}}\right)}$

The plots of the $\mathrm{sf}$ and $\mathrm{hf}$ of the TE-WD are respectively shown in figure 3 and 4 for selected values $\lambda=a, \gamma=b, \theta=c$, and $k=d$.

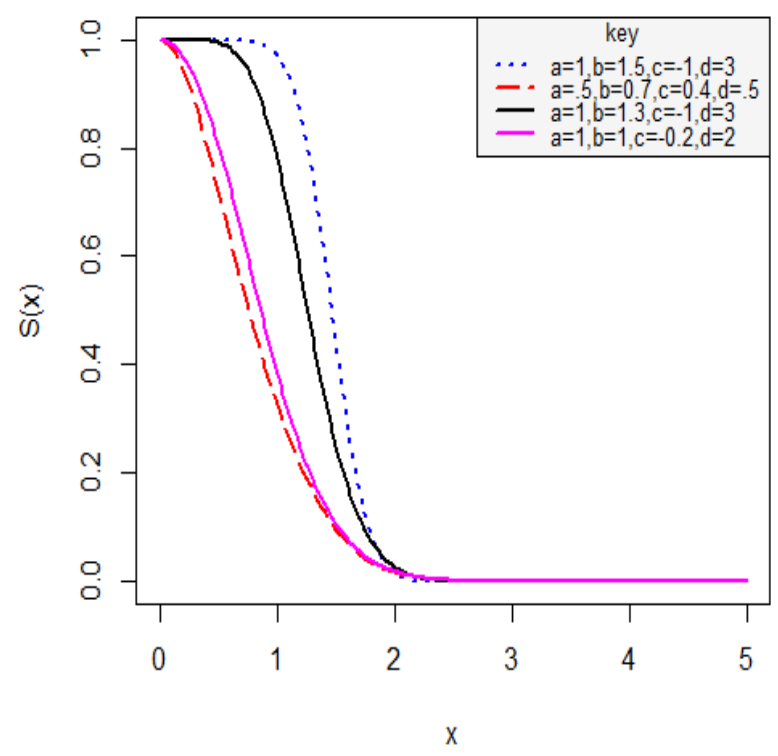

Figure 3: sf of TE-WD

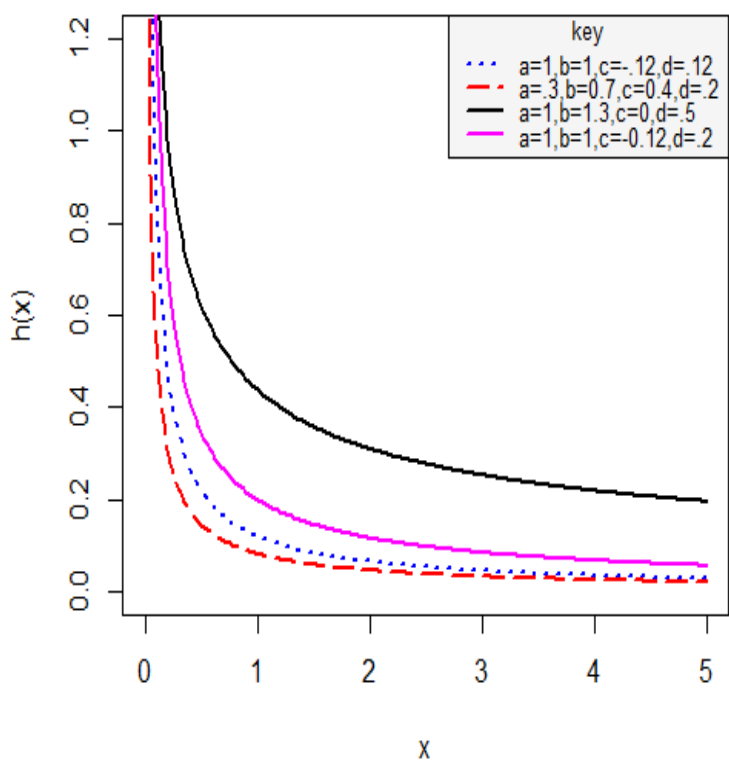

Figure 4: hf of TE-WD

Quantile Function of TE- W Distribution

Definition 3. For a nonnegative continuous random variable say $X$ that follows the TE-WD, the quantile function is given by;

$$
x_{u}=\gamma\left(\ln \left(\frac{(\theta-1)+\sqrt{(\theta-1)^{2}+4 \theta(1-u)}}{2 \theta}\right)^{-\frac{1}{\lambda}}\right)^{\frac{1}{k}} \quad \text { for } \theta \neq 0
$$

A simulation study was used to evaluate the behavior of skewness, kurtosis, mean and variance of the TE-W model. The results are given in table 1 for some assumed values of the distribution parameters. The results has shown that the skewness, kurtosis, mean and variance decreases as the values of the shape parameter $(k)$ increase. 
Table 1: Skewness, kurtosis, average and variance for some random choices of the parameter values PARAMETERS $\lambda=2, \gamma=1, \theta=0.2$ $\lambda=2, \gamma=1, \theta=0.2$

\begin{tabular}{c|ccccc}
\hline $\boldsymbol{k} \downarrow$ & Skewness & Kurtosis & $\boldsymbol{k} \downarrow$ & Mean & Variance \\
& & & & & 26.6025 \\
$\mathbf{0 . 4}$ & 5.0529 & 48.1953 & $\mathbf{0 . 4}$ & 6.7915 & 2.1405 \\
$\mathbf{0 . 6}$ & 3.0253 & 19.4309 & $\mathbf{0 . 6}$ & 3.4511 & 0.5452 \\
$\mathbf{1 . 0}$ & 2.3718 & 12.5862 & $\mathbf{0 . 8}$ & 2.4994 & 0.2206 \\
$\mathbf{1 . 2}$ & 2.0635 & 9.8870 & $\mathbf{1 . 0}$ & 2.0687 & 0.1136 \\
$\mathbf{1 . 4}$ & 1.8859 & 8.5028 & $\mathbf{1 . 2}$ & 1.8269 & 0.0676 \\
$\mathbf{1 . 6}$ & 1.7707 & 7.6754 & $\mathbf{1 . 4}$ & 1.6730 & 0.0443 \\
\hline
\end{tabular}

Entropy and Order Statistic of the distribution

In this section, we discuss the Renyi entropy and order statistics

\section{Renyi Entropy of the TE-WD}

The Renyi Entropy of a random variable say X having Transmuted Exponential- Weibull Distribution is given by;

$I_{R}(\rho)=\frac{1}{1-\rho} \log \left\{\left(\frac{\lambda k}{\gamma}\right)^{\rho}\left(\frac{x}{\gamma}\right)^{\rho(k-1)} \sum_{j, m=0}^{\infty} w_{j, m, \rho} \frac{1}{k}\left(\frac{\lambda}{\gamma^{k}}(\rho+m)\right)^{-\left(\frac{\rho(k-1)+1}{k}\right)} \Gamma\left(\frac{\rho(k-1)+1}{k}\right)\right\}$

Where,

$w_{j, m, \rho}=\frac{(-1)^{j+m} \Gamma(\rho+1) \Gamma(j+1) \theta^{j} 2^{m}}{j ! m ! \Gamma(\rho+1-j) \Gamma(j+1-m)}$

\section{Order Statistic of the model}

The density function $(\mathrm{df})$ of the jth order statistics for a given random samples $X_{1}, X_{2}, \ldots, X_{n}$ from the cdf and of TE-WD is given as;

$f_{j: n}(x)=\frac{n ! \lambda k}{(j-1) !(n-j) ! \gamma^{k}}\left((1-\theta)+2 \theta e^{-\lambda\left(\frac{x}{\gamma}\right)^{k}}\right) m_{c, d, f g, q}$

Where,

$$
m_{c, d, f g, q}=\sum_{c, d, f g, q=0}^{\infty} \frac{(-1)^{c+f+g+q} \Gamma j \Gamma j \Gamma(n-j+1) \Gamma(f+1) \theta^{d+f}\left(\frac{\lambda}{\gamma^{k}}(1+c+d+g+n-j)\right)^{q} x^{q k+k-1}}{c ! d ! f ! g ! q ! \Gamma(j-c) \Gamma(j-d) \Gamma(n-j+1-f) \Gamma(f+1-g)}
$$

\section{Estimation of Parameters of the Transmuted Exponential -Weibull Distribution}

The estimation of the TE-WD parameters is achieved by using the maximum likelihood estimation technique. Let $x_{1}, x_{2}, \ldots, x_{n}$ be a random sample from the $T E-W$ Distribution with unknown parameter vector $\psi=(\lambda, k, \gamma, \theta)^{T}$. For determining the MLE of $\psi$, we have the log-likelihood function:

$$
L(\psi)=n \log \left(\frac{\lambda k}{\gamma}\right)+\sum_{i=1}^{n} \log \left(\frac{x_{i}}{\gamma}\right)^{k-1}-\lambda \sum_{i=1}^{n}\left(\frac{x_{i}}{\gamma}\right)^{k}+\sum_{i=1}^{n} \log \left(1-\theta+2 \theta e^{-\lambda\left(\frac{x_{i}}{\gamma}\right)^{k}}\right)
$$




$$
\begin{aligned}
& L(\psi)=n \log \lambda+n \log k-n \log \gamma-\lambda \sum_{i=1}^{n}\left(\frac{x_{i}}{\gamma}\right)^{k}+(k-1) \sum_{i=1}^{n} \log x_{i}-(k-1) \sum_{i=1}^{n} \log \gamma+\sum_{i=1}^{n} \log \left(1-\theta+2 \theta e^{-\lambda\left(\frac{x_{i}}{\gamma}\right)^{k}}\right) \\
& L(\psi)=n \log \lambda+n \log k-n k \log \gamma-\lambda \sum_{i=1}^{n}\left(\frac{x_{i}}{\gamma}\right)^{k}+(k-1) \sum_{i=1}^{n} \log x_{i}+\sum_{i=1}^{n} \log \left(1-\theta+2 \theta e^{-\lambda\left(\frac{x_{i}}{\gamma}\right)^{k}}\right) \\
& U_{\lambda}=\frac{\delta L(\psi)}{\delta \lambda}=\frac{n}{\lambda}-\sum_{i=1}^{n}\left(\frac{x_{i}}{\gamma}\right)^{k}-2 \theta \sum_{i=1}^{n} \frac{\left(\frac{x_{i}}{\gamma}\right)^{k} e^{-\lambda\left(\frac{x_{i}}{\gamma}\right)^{k}}\left(1-\theta+2 \theta e^{\left.-\lambda\left(\frac{x_{i}}{\gamma}\right)^{k}\right)}\right)}{(1-\theta)}
\end{aligned}
$$$$
U_{k}=\frac{\delta L(\psi)}{\delta k}=\frac{n}{k}-n \log \gamma-\lambda \sum_{i=1}^{n}\left(\frac{x_{i}}{\gamma}\right)^{k} \ln \left(\frac{x_{i}}{\gamma}\right)+\sum_{i=1}^{n} \log x_{i}-2 \theta \lambda \sum_{i=1}^{n} \frac{e^{-\lambda\left(\frac{x}{\gamma}\right)^{k}}\left(\frac{x}{\gamma}\right)^{k} \ln \left(\frac{x}{\gamma}\right)}{\left(1-\theta+2 \theta e^{\left.-\lambda\left(\frac{x_{i}}{\gamma}\right)^{k}\right)}\right.}
$$$$
U_{\gamma}=\frac{\delta L(\psi)}{\delta \gamma}=\frac{-n k}{\gamma}+\lambda \sum_{i=1}^{n} x_{i}^{k} \gamma^{-(k+1)}-2 \theta \lambda k \gamma \sum_{i=1}^{n} \frac{x^{k} \gamma^{-(k+1)} e^{-\lambda\left(\frac{x}{\gamma}\right)^{k}}}{\left.1-\theta+2 \theta e^{-\lambda\left(\frac{x_{i}}{\gamma}\right)^{k}}\right)}
$$$$
U_{\theta}=\frac{\delta L(\psi)}{\delta \theta}=\sum_{i=1}^{n} \frac{2 e^{-\lambda\left(\frac{x}{\gamma}\right)^{k}}-1}{\left(1-\theta+2 \theta e^{-\lambda\left(\frac{x_{i}}{\gamma}\right)^{k}}\right)}
$$

Setting and solving the nonlinear system of equations $U_{\lambda}=U_{k}=U_{\gamma}=U_{\theta}=0$ at the same time yields the MLE $\widehat{\psi}=(\hat{\lambda}, \hat{k}, \hat{\gamma}, \widehat{\theta})^{T}$. Generally, it is more practical to use nonlinear methods of optimization such as the Newton-Rapson algorithm to maximize $\mathrm{L}$ numerically in order to solve certain equations.

\section{Simulation Study}

Within this portion, a simulation analysis is performed to assess the efficiency of the MLEs of the TEWD parameters. 
Table 2: Mean values of the MLEs, Biases and MSEs of the TEWD for $\lambda=1, k=3, \gamma=0.2, \theta=1$

\begin{tabular}{|c|c|c|c|c|}
\hline $\mathrm{n}$ & Parameter & Estimate & Bias & MSE \\
\hline \multirow{4}{*}{$\mathrm{n}=20$} & $\lambda$ & 1.0263 & 0.0263 & 0.0117 \\
\hline & $\boldsymbol{k}$ & 3.2935 & 0.2935 & 0.4625 \\
\hline & $\gamma$ & 0.1932 & -0.0067 & 0.0003 \\
\hline & $\boldsymbol{\theta}$ & 0.9101 & -0.0898 & 0.0385 \\
\hline \multirow{4}{*}{$\mathrm{n}=50$} & $\lambda$ & 1.0211 & 0.0211 & 0.0101 \\
\hline & $\boldsymbol{k}$ & 3.1402 & 0.1402 & 0.1553 \\
\hline & $\gamma$ & 0.1948 & -0.0052 & 0.0002 \\
\hline & $\boldsymbol{\theta}$ & 0.9162 & -0.0838 & 0.0322 \\
\hline \multirow{4}{*}{$\mathrm{n}=100$} & $\lambda$ & 1.0260 & 0.0260 & 0.0108 \\
\hline & $\boldsymbol{k}$ & 3.0904 & 0.0904 & 0.0805 \\
\hline & $\gamma$ & 0.1960 & -0.0040 & 0.0001 \\
\hline & $\theta$ & 0.9139 & -0.0861 & 0.0327 \\
\hline \multirow{4}{*}{$\mathrm{n}=150$} & $\lambda$ & 1.0263 & 0.0263 & 0.0100 \\
\hline & $\boldsymbol{k}$ & 3.0707 & 0.0707 & 0.0502 \\
\hline & $\gamma$ & 0.1965 & -0.0035 & $9.6202 \mathrm{e}-05$ \\
\hline & $\theta$ & 0.9202 & -0.0798 & 0.0292 \\
\hline \multirow{4}{*}{$\mathrm{n}=200$} & $\lambda$ & 1.0236 & 0.0236 & 0.0167 \\
\hline & $\boldsymbol{k}$ & 3.0674 & 0.0674 & 0.0415 \\
\hline & $\gamma$ & 0.1966 & -0.0034 & 0.0001 \\
\hline & $\boldsymbol{\theta}$ & 0.9221 & -0.0779 & 0.0296 \\
\hline
\end{tabular}

\section{DISCUSSION OF THE SIMULATION RESULTS}

1000 samples of size, $n=20,50,100,150$ and 200 of the TEWD for fixed choice of parameters for $\lambda=1, k=3, \gamma=$ $0.2, \theta=1$ were generated. The evaluation of estimates is based on the mean of the MLEs of the model parameters, bias and the mean squared error (MSE) of the MLEs. The empirical study was conducted using the programming language $\mathrm{R}$, and the results are shown in Table 2 . The values in the table indicate that the estimates for these sample sizes are very stable and, more importantly, the estimates are close to the true values. In addition, from Table 2 the biases and MSEs are decreasing as $n$ increases.

\section{APPLICATIONS}

Here, a real-life time dataset to evaluate the goodness-of-fit of TE-WD were provided.

\section{Real dataset}

The data set represent the failure times of 50 components (per $1000 \mathrm{~h}$ ). For previous studies with the data sets see (Aryal and Elbatal 2015). The observations are:

0.036, 0.058, 0.061, 0.074, 0.078, 0.086, 0.102, 0.103, 0.114,
$0.116,0.148,0.183,0.192,0.254,0.262,0.379,0.381,0.538$, $0.570,0.574,0.590,0.618,0.645,0.961,1.228,1.600,2.006$, 2.054, 2.804, 3.058, 3.076, 3.147, 3.625, 3.704, 3.931, 4.073, $4.393,4.534,4.893,6.274,6.816,7.896,7.904,8.022,9.337$, $10.940,11.020,13.880,14.730,15.080$.

We equally used this dataset to compare the TE-W model with Beta Weibull (BW), Exponentiated Generalized Weibull (EGW) distribution and Log Gamma I Weibull (LGW).

In order to determine the best out of the competing models, we will make use of some criteria including $A I C$ (Akaike Information Criterion), $C A I C$ (Consistent Akaike Information Criterion), HQIC (Hannan-Quinn Information Criteria) and $B I C$ (Bayesian Information Criterion). These criteria are mathematically expressed as:

$\mathrm{AIC}=-2 \mathrm{~L}+2 \mathrm{k}, \mathrm{CAIC}=-2 \mathrm{~L}+2 \mathrm{kn} /(\mathrm{n}-\mathrm{k}-1), \mathrm{HQIC}=-2 \mathrm{~L}$ $+2 \mathrm{k} \log (\log (\mathrm{n}))$ and $\mathrm{BIC}=-2 \mathrm{~L}+\mathrm{k} \log (\mathrm{n})$

Where $\mathrm{L}$ stands for the log-likelihood function, $\mathrm{k}$ is the number of parameters of the model and $\mathrm{n}$ represents the sample size.

We also evaluate other measures such as Anderson- Darling $\left(\mathrm{A}^{*}\right)$ and Cramer- Von Mises $\left(\mathrm{W}^{*}\right)$ Statistics.

Note: Among competing models, the model with the lowest value of these measures is considered to be the best 
Table 3: Gives the summary statistics of failure time data

\begin{tabular}{cccccccc}
\hline n & Min. & Median & Mean & Variance & Max. & Skewness & Kurtosis \\
\hline 50 & 0.0360 & 1.414 & 3.3430 & 17.4847 & 15.08 & 1.4167 & 4.0846 \\
\hline
\end{tabular}

Table 4: Estimated parameters for failure time data

\begin{tabular}{|c|c|c|c|c|c|c|c|}
\hline Model & $\hat{\lambda}$ & $\widehat{\theta}$ & $\widehat{a}$ & l & $\hat{k}$ & $\widehat{k}$ & $\hat{\gamma}$ \\
\hline TE-W & 0.9056 & 0.1006 & - & - & 0.6736 & 2.3725 & \\
\hline EGW & - & - & 0.3792 & 0.7847 & 0.7705 & 0.9792 & \\
\hline BW & - & - & 0.7608 & 0.6645 & 0.7808 & 2.0396 & \\
\hline LGW & - & - & 1.4794 & 1.0419 & 0.5189 & 1.1099 & \\
\hline
\end{tabular}

\begin{tabular}{cccccc} 
& \multicolumn{5}{c}{ Table 5: Goodness-of-fit statistics for the dataset } \\
\hline Model & -LL & AIC & CAIC & HQIC & BIC \\
& & & & & \\
\hline TE-W & $\mathbf{1 0 2 . 3 3 4 9}$ & $\mathbf{2 1 2 . 6 6 9 7}$ & $\mathbf{2 1 3 . 5 5 8 6}$ & $\mathbf{2 1 5 . 5 8 2 2}$ & $\mathbf{2 2 0 . 3 1 7 8}$ \\
EGW & 102.3563 & 212.7125 & 213.6014 & 215.6249 & 220.3606 \\
BW & 102.3489 & 212.6978 & 213.5867 & 215.6102 & 220.3459 \\
LGW & 102.3479 & 212.6958 & 213.5847 & 215.6082 & 220.3439 \\
\hline
\end{tabular}

Table 6: Goodness-of-fit statistics for failure time data

\begin{tabular}{cll}
\hline Model & \multicolumn{1}{c}{$\mathrm{W}^{*}$} & \multicolumn{1}{c}{$\mathrm{A}^{*}$} \\
\hline TE-W & $\mathbf{0 . 1 4 8 0}$ & $\mathbf{0 . 9 2 8 8}$ \\
EGW & 0.1499 & 0.9459 \\
BW & 0.1492 & 0.9435 \\
LGW & 18.17101 & 100.2162 \\
\hline
\end{tabular}

\section{DISCUSSION OF THE RESULTS}

Table 3 provides the descriptive statistics of the real datase while in Table 4, the estimates of the parameters for the TE-W, Beta Weibull (BW), Exponentiated Generalized Weibull (EGW) distribution, and Log Gamma I Weibull (LGW) models were provided.

Tables 5 and 6 show that the smallest values of the goodnessof-fit statistics are found in the Transmuted Exponential Weibull (TE-W) as compared to other existing distributions in the literature (Beta Weibull (BW), Exponentiated Generalized Weibull (EGW) distribution, and Log Gamma I Weibull (LGW) distribution). The Transmuted Exponential-Weibull model, therefore, was seen as the strongest among the competing distributions.

\section{CONCLUSION}

In this study, a new four-parameter distribution called Transmuted Exponential-Weibull Distribution is presented. An explicit expression for some of its mathematical and structural properties is derived and presented. The TE-WD's usefulness and ability is exemplified by applications to the failure time dataset. The comparison is based on fit statistics such as AIC, BIC, CAIC HQIC, Cramer von-Mises and Anderson-Darling for goodness. The findings of goodness-of-fit statistics show that the TE-W model provides better fit than other competing models. Further research can look at the estimation of confidence intervals for the parameters of the proposed model.

\section{ACKNOWLEDGEMENTS}

The authors wish to thank the referees for their suggestions and insightful remarks which enriched the article greatly. This research did not receive any specific grant from funding agencies in the public or commercial sector.

\section{REFERENCES}

Ahmad, A., Ahmad, S., \& Ahmed, A. (2017). Characterization and Estimation of Weibull-Rayleigh distribution with Applications to life time data. Appl. Math. Inf. Sci. Lett, 5, 7179.

Alzaatreh, A., Lee, C., and Famoye, F. (2013). A new method for generating families of continuous distributions. Metron, 71, 63-79.

Aryal, G. R., \& Tsokos, C. P. (2011). Transmuted Weibull distribution: A generalization of theWeibull probability distribution. European Journal of Pure and Applied Mathematics, 4(2), 89-102.

Aryal, G., \& Elbatal, I. (2015). On the exponentiated generalized modified Weibull distribution. Communications for Statistical Applications and Methods, 22(4), 333-348.

Bourguignon, M., Silva, R.B. and Cordeiro, G.M. (2014). The Weibull-G Family of Probability Distributions. Journal of Data Science, 12, 53-68.

Calderín-Ojeda, E. (2015). On the Composite Weibull-Burr Model to describe claim data. Communications in Statistics: Case Studies, Data Analysis and Applications, 1(1), 59-69.

Cordeiro, G. M., Afify, A. Z., Yousof, H. M., Cakmakyapan, S., \& Ozel, G. (2018). The Lindley Weibull distribution: properties and applications. Anais da Academia Brasileira de Ciências, 
90(3), 2579-2598.

Cordeiro, G. M., Hashimoto, E. M., \& Ortega, E. M. (2014). The McDonald Weibull model. Statistics, 48(2), 256-278.

Cordeiro, G.M., Ortega, E.M.M. and Da Cunha, D.C.C. (2013). The exponentiated generalized class of distribution. Journal of Data Science, 11, 1-27.

Cordeiro, G.M., Ortega, E.M.M., Popovic, B.V. and Pescim, R.R. (2014). The Lomax generator of distributions: Properties, minification process and regression model. Applied Mathematics and Computation, 247, 465-486.

Daniyal, M., \& Aleem, M. (2014). On the mixture of Burr XII and Weibull distributions. Journal of Statistics Applications \& Probability, 3(2), 251.

Mead, M.E., Cordeiro, G.M., Afify, A., Al-Mofleh, H. (2019). The Alpha Power Transformation Family: Properties and Applications. Pakistan Journal of Statistics and Operation Research, 15(3), 525-545.

Mohammed, A. S. (2019). Theoretical Analysis of the Exponentiated Transmuted Kumaraswamy Distribution with Application. Annals of Statistical Theory and Applications (ASTA), 1, 51-60.

Mohammed, A. S., \& Ugwuowo, F. I. (2020). A NEW FAMILY OF DISTRIBUTIONS FOR GENERATING SKEWED MODELS: PROPERTIES AND APPLICATIONS. Pakistan Journal of Statistics, 36(2).

Mohammed, A. S., \& Yahaya, A. (2019). Exponentiated Transmuted Inverse Exponential Distribution with Application. Annals of Statistical Theory and Applications (ASTA), 2, 71-80.
Mudholkar, G. S., Srivastava, D. K., \& Kollia, G. D. (1996). A generalization of the Weibull distribution with application to the analysis of survival data. Journal of the American Statistical Association, 91(436), 1575-1583.

Murthy, D. N., Xie, M., and Jiang, R. (2004). Weibull Models.

John Wiley and Sons, New Jersey.

Shanker R, Fesshaye H, Selvaraj S (2015) On Modeling of Lifetimes Data Using Exponential and Lindley Distributions. Biom Biostat Int J 2(5): 00042. DOI: 10.15406/bbij.2015.02.00042

Teamah, A. E. A., Elbanna, A. A., \& Gemeay, A. M. (2020). FRÉCHET-WEIBULL DISTRIBUTION WITH APPLICATIONS TO EARTHQUAKES DATA SETS. Pakistan Journal of Statistics, 36(2).

Xie M., Tang, Y. \& Goh, T. N. (2002). A modified Weibull extension with bathtub failure rate function. Reliab Eng Syst Saf 76: 279-285.

Xie, M. \& lai, C. D. (1995). Reliability analysis using an additive Weibull model with bathtubshaped failure ratefunction. Reliab Eng Syst Saf 52: 87-93.

Zaindin, M., \& Sarhan, A. M. (2009). Parameters estimation of the modified Weibull distribution. Applied Mathematical Sciences, 3(11), 541-550. 